\title{
Manifestações orais nas doenças mieloproliferativas: Uma revisão de literatura
}

\author{
Oral manifestations in myeloproliferative diseases: A literature review \\ Manifestaciones orales en enfermedades mieloproliferativas: Revisión de la literatura
}

Hélio Mateus Silva Nascimento ORCID: https://orcid.org/0000-0003-1551-8139

Universidade Federal do Delta do Parnaíba, Brasil

E-mail: helio_mateus_@hotmail.com

Even Herlany Pereira Alves

ORCID: https://orcid.org/0000-0001-7566-1282

Universidade Federal do Delta do Parnaíba, Brasil

E-mail: even.herlany@gmail.com

Wesley Rodrigues da Silva

ORCID: https://orcid.org/0000-0002-2241-5843

Universidade Federal do Delta do Parnaíba, Brasil

E-mail:mr.wesleyrodrigues@gmail.com

John Arlley Sousa Pinho de Lira

ORCID: https://orcid.org/0000-0001-6565-6260

Universidade Federal do Delta do Parnaíba, Brasil

E-mail: arlley_pinho@hotmail.com

Francisco Alex da Rocha Coelho

ORCID: https://orcid.org/0000-0002-3308-375X

Universidade Federal do Delta do Parnaíba, Brasil

E-mail: alex123rocha@hotmail.com

Paulo Roberto Carneiro Gomes

ORCID: https://orcid.org/0000-0002-2911-7785

Universidade Federal do Delta do Parnaíba, Brasil

E-mail: paulo.c.gomes1@outlook.com

Julyanne Maria Saraiva de Sousa

ORCID: https://orcid.org/0000-0001-9041-0860

Universidade Federal do Delta do Parnaíba, Brasil

E-mail: jully.yanne@ gmail.com

Mateus Cardoso do Amaral

ORCID: https://orcid.org/0000-0003-2735-9892

Universidade Federal do Delta do Parnaíba, Brasil

E-mail: mateuscamaral@gmail.com

Ayane Araújo Rodrigues

ORCID: https://orcid.org/0000-0001-7792-6993

Universidade Federal do Delta do Parnaíba, Brasil

E-mail: ayanerodrigues2012@ hotmail.com

André dos Santos Carvalho

ORCID: https://orcid.org/0000-0001-5259-6481

Universidade Federal do Delta do Parnaíba, Brasil

E-mail: fko.andre@gmail.com

Thais Amanda de Lima Nunes

ORCID: https://orcid.org/0000-0002-1924-4828

Universidade Federal do Delta do Parnaíba, Brasil

E-mail: amandalima-@hotmail.com.br

Samara Marques de Oliveira

ORCID: https://orcid.org/0000-0003-0034-5685

Universidade Federal do Delta do Parnaíba, Brasil

E-mail: samaramarques1806@gmail.com

Rubens Renato de Sousa Carmo

ORCID: https://orcid.org/0000-0001-7135-7531

Universidade Federal do Delta do Parnaíba, Brasil

E-mail: rubensrenatobezerra@gmail.com

Daniel Fernando Pereira Vasconcelos

ORCID: https://orcid.org/0000-0002-3331-452X

Universidade Federal do Delta do Parnaíba, Brasil

E-mail: prof.dr.daniel.vasconcelos@gmail.com 


\begin{abstract}
Resumo
Objetivo: Abordar as manifestações causadas pelas alterações provenientes das diversas doenças mieloproliferativa na cavidade oral e as implicações na vida do paciente, de maneira a fornecer embasamento teórico que ajude no diagnóstico precoce e terapia dessas patologias. Materiais e Métodos: trata-se de uma revisão de literatura onde foi realizada uma busca nos bancos de dados relacionados às áreas da Saúde como: SCIELO, PUBMED e LILACS usando corte temporal de 2016 a 2020, com os descritores "hematologia" ou "doenças hematológicas"; "afecção" ou "manifestações orais" e "leucemia" ou "alterações hematologicas". Discussão: O presente trabalho está relacionado as alterações orais causadas pelas doenças mieloproliferativas, onde poucos são os relatos destas alterações na literatura, uma vez que não se tinha o conhecimento do acometimento da cavidade oral com as doenças, em busca de aprimorar o diagnostico diferencial este trabalho trás as principais alterações encontradas na literatura sobre as alterações bucais. Conclusão: Com isso é evidente a necessidade de acompanhamento multiprofissional dos pacientes acometidos por estas enfermidades e espera-se que esta pesquisa juntamente com pesquisas futuras, possa esclarecer as alterações provocadas pelas doenças mieloproliferativas, principalmente suas manifestações na cavidade oral, para um diagnostico diferencial e precoce. Dessa forma, concluímos que há uma relação direta das alterações clínicas na cavidade oral em indivíduos com doença mieloproliferativa.
\end{abstract}

Palavras-chave: Diagnóstico; Doenças hematológicas; Hematologia; Leucemias.

\begin{abstract}
Objective: to address the manifestations caused by the alterations caused by the various myeloproliferative diseases in the oral cavity and the implications in the patient's life, in order to provide a theoretical basis that helps in the early diagnosis and therapy of these pathologies. Materials and Methods: this is a literature review where a search was carried out in the databases related to the areas of Health such as: SCIELO, PUBMED and LILACS using time cut from 2016 to 2020, with the descriptors "hematology" or "diseases hematological "; "affection" or "oral manifestations" and "leukemia" or "hematological changes". Discussion: The present work is related to oral changes caused by myeloproliferative diseases, where there are few reports of these changes in the literature, since there was no knowledge of the involvement of the oral cavity with diseases, in an attempt to improve this differential diagnosis. This work brings the main alterations found in the literature on oral alterations. Conclusion: With this, the need for multidisciplinary monitoring of patients affected by these diseases is evident and it is hoped that this research, together with future research, can clarify the changes caused by myeloproliferative diseases, especially their manifestations in the oral cavity, for a differential diagnosis and precocious. Thus, we conclude that there is a direct relationship between clinical changes in the oral cavity in individuals with myeloproliferative disease.
\end{abstract}

Keywords: Diagnosis; Hematological diseases; Hematology; Leukemia.

\title{
Resumen
}

Objetivo: abordar las manifestaciones provocadas por las alteraciones provocadas por las distintas enfermedades mieloproliferativas en la cavidad bucal y las implicaciones en la vida del paciente, con el fin de aportar una base teórica que ayude en el diagnóstico y tratamiento precoz de estas patologías. Material y Métodos: se trata de una revisión de la literatura donde se realizó una búsqueda en las bases de datos relacionadas con las áreas de la Salud tales como: SCIELO, PUBMED y LILACS utilizando corte de tiempo de 2016 a 2020, con los descriptores "hematología" o "enfermedades hematológicas". "; "afección" o "manifestaciones orales" y "leucemia" o "alteraciones hematológicas". Discusión: El presente trabajo se relaciona con los cambios bucales provocados por enfermedades mieloproliferativas, donde existen pocos reportes de estos cambios en la literatura, ya que no se tenía conocimiento de la afectación de la cavidad oral con enfermedades, en un intento por mejorar este diagnóstico diferencial. Este trabajo trae las principales alteraciones encontradas en la literatura sobre alteraciones bucales. Conclusión: Con esto, se hace evidente la necesidad de un seguimiento multiprofesional de los pacientes afectados por estas enfermedades y se espera que esta investigación, junto con investigaciones futuras, pueda esclarecer los cambios provocados por las enfermedades mieloproliferativas, especialmente sus manifestaciones en la cavidad bucal, para un diagnóstico diferencial y precoz. Por lo tanto, concluimos que existe una relación directa entre los cambios clínicos en la cavidad oral en individuos con enfermedad mieloproliferativa.

Palabras clave: Diagnóstico; Enfermedades hematológicas; Hematología; Leucemias.

\section{Introdução}

Por ser a janela para a saúde geral de um paciente a cavidade oral é considerada como a intersecção da medicina com a odontologia. Onde centenas de doenças e medicamentos podem afetar suas estruturas, e as condições patológicas na boca têm um impacto sistêmico maior do que muitos imaginam (Kane, 2017). Estudos já mostraram a relação da associação entre doença periodontal e certas outras condições sistêmicas, incluindo doença vascular aterosclerótica, doença pulmonar, diabetes, 
complicações relacionadas à gravidez, esteatose e doença renal (Carvalho et al., 2017; França et al., 2017; Kane, 2017; Gomes et al., 2020).

Através do sangue e sistema linfático os tecidos dos nossos diversos órgãos têm relação direta com o tecido oral, sendo uma constante conexão. Com isso o equilíbrio da saúde oral é influenciado diretamente por alterações sistêmicas, levando ao aumento da frequência das manifestações da cavidade oral, sendo o primeiro sinal de um desequilibro sistêmico, onde é crucial o exame da cavidade oral pois ele pode denunciar essas alterações (Oliveira et al., 2015).

As doenças mieloproliferativas são classificadas como alterações não fisiológicas de células mielóide no sistema hematopoiético, levando a uma superprodução de células sanguíneas mieloides maduras e funcionais, estas alterações podem ser devido a mutações somáticas que causam uma ativação constitutiva de vias de sinalização fisiológica em células tronco hematopoiéticas e progenitora, levando a uma expansão clonal de células progenitoras mieloides e hiperplasia de uma ou multilinhagem (Braun et al., 2020; Eichstaedt et al., 2020).

Diversas são as alterações sistêmicas provocadas pelas doenças mieloproliferativas, onde consequentemente afeta as estruturas da cavidade oral, devido esta grande importância das doenças hematológicas, este estudo objetiva abordar as manifestações causadas pelas alterações provenientes das diversas doenças mieloproliferativa na cavidade oral e as implicações na vida do paciente, de maneira a fornecer embasamento teórico para que ajude no diagnóstico precoce e terapia dessas patologias.

\section{Metodologia}

Este estudo trata-se de uma revisão de literatura de caráter qualitativo de acordo com Estrela (2018), onde foi realizada uma busca nos bancos de dados relacionados às áreas da Saúde como: SCIELO, PUBMED e LILACS.

\subsection{Critério de Inclusão}

Os artigos incluídos neste estudo devem obedecer aos seguintes critérios: (1) estudos publicados gratuitamente na integra; (2) Ensaios clínicos randomizados; (3) Relatos de caso sobre a relação das doenças mieloproliferativas e manifestações orais e (4) estudos casos controles. Estudos que não forneceram informações suficientes ou não atender a qualquer um desses critérios foram excluídos.

\subsection{Estratégia de Busca}

Uma busca sistemática da literatura foi realizada nas bases de dados científicas para estudos publicados entre 2016 a 2020 que abordavam temas relacionados. As seguintes palavras-chave foram utilizadas na busca nos bancos de dados: ("hematologia" ou "Doenças hematológicas"); ("afecção" ou "manifestações orais") e ("leucemia" ou "alterações hematologicas") em inglês e português. Não houve restrições no idioma da pesquisa e todas as citações dos estudos foram analisadas para identificar possíveis estudos que se enquadravam na busca.

\section{Revisão da Literatura}

O termo "Distúrbios Mieloproliferativos" descreve alterações não fisiológicas do compartimento mielóide no sistema hematopoiético levando a uma superprodução de células sanguíneas mieloides maduras e funcionais (Braun \& Zeiser, 2020). A classificação da Organização Mundial da Saúde (OMS) para tumores hematopoiéticos e Neoplasias Mieloproliferativas (MPN) distingue diferentes formas da doença em leucemia mielóide crônica, leucemia neutrófila crônica, 
leucemia eosinofílica crônica, mielofibrose idiopática crônica (MIC), policitemia vera (PV), trombocitopenia essencial (ET) e MPN não classificada (MPN-U) (Barbui et al., 2018; Braun \& Zeiser, 2020).

As três formas clássicas de MPN BCR-ABL-negativas, que são os transtornos mais frequentes entre todas as doenças mieloproliferativas, que compreendem PV, MIC e ET. Mutações somáticas causam uma ativação constitutiva de vias de sinalização fisiológica em células tronco hematopoiéticas e progenitoras, levando a uma expansão clonal de células progenitoras mieloides e hiperplasia de uma ou multilinearagem (Spivak, 2017; Vainchenker \& Kralovics, 2017). As MPNs são caracterizadas pelo aumento da produção de células sanguíneas totalmente diferenciadas e completamente funcionais da linhagem mielóide. ET, PV e MIC são clinicamente classificados por uma superprodução de plaquetas funcionais, aumento do número de glóbulos vermelhos e alta contagem de glóbulos brancos e fibrose de medula óssea e baço, respectivamente (Vainchenker \& Kralovics, 2017; Braun \& Zeiser, 2020).

\subsection{Leucemia Mieloide Crônica (LMC)}

É um câncer de sangue causado pelo BCR-ABL1, que é um gene híbrido que surge quando o DNA do gene BCR no cromossomo 22 e do gene ABL1 no cromossomo 9 quebra e recombinam-se, em uma célula com a capacidade biológica, intrínseca ou adquirida, de causar leucemia. BCR-ABL1 codifica uma proteína quimerica de 210 KD (P210 ${ }^{\text {BCRABL1}}$ ), com atividade constitutiva de tirosina-quinase (Zhao \& Deininger, 2020; Houshmand et al., 2020).

Por vários mecanismos indefinidos, essa anormalidade resulta na expansão do clone de células mieloides. A célula em que o BCR-ABL1 ocorre pela primeira vez e que causa a LMC é chamada de célula-tronco leucêmica. Algumas progêneres desta célula também podem ter ou adquirir características de células-tronco, incluindo a capacidade biológica de causar recidiva, esta doença possui uma incidência de 1 a 2 casos por 100 mil adultos. É responsável por aproximadamente 15\% dos casos recém-diagnosticados de leucemia em adultos de acordo com a Sociedade Americana do Câncer (Jabbour \& Kantarjian, 2018; Zhao \& Deininger, 2020).

Onde aproximadamente 50\% dos pacientes diagnosticados com LMC nos Estados Unidos não apresentam sintomas característicos, onde são frequentemente diagnosticados durante um exame físico de rotina ou exames de sangue (Jabbour \& Kantarjian, 2018).

Os sintomas mais comuns são: fadiga, perda de peso, mal-estar, fácil saciedade e dor do quadrante superior esquerdo (Melo \& Gonçalves, 2016; Lago \& Petroni, 2017). Suas manifestações raras incluem sangramento, trombose, artrite gouty, priapismo, hemorragias na retina, e ulceração gastrointestinal superior e sangramento (Sossela et al., 2017; Jabbour \& Kantarjian, 2018; Ribeiro et al., 2019).

Quando falamos de alterações orais nesta patologia destacam-se: queilite angular, manchas violáceas, pápula sobre o freio labial, hiperplasia fibrosa inflamatória, úlcera sublingual, papila incisiva hiperemiada, múltiplas petéquias e descamação labial, fazendo necessário o acompanhamento por profissionais dentistas dos pacientes (Boddu et al., 2017; Shimizu et al., 2017; Ratre et al., 2018; Shen et al., 2018; Tag-Adeen et al., 2018).

\subsection{Leucemia Neutrofilica Crônica (LNC)}

A LNC é uma rara síndrome mieloproliferativa crônica, onde poucas são os casos relatados na literatura. Em sua maioria em pessoas com mais de 50 anos, contudo há relatos também em jovens entre 15 e 29 anos (Maxson \& Tyner, 2017; Ouyang et al., 2017).

Seu diagnóstico é difícil de distinguir entre a relação entre a LMC, onde um estudo citogenético é de crucial importância para a diferenciação entre LNC e LMC (Szuber et al., 2020). 
Por ser uma doença caracterizada por neutrofilia persistente no sangue periférico, medula óssea hipercelular com hiperplasia da série granulocítica, principalmente madura. Suas manifestações clínicas incluem hepatoesplenomegalia que não é acompanhada por episódios febris. Onde normalmente afeta pacientes de ambos os sexos, alterando assim os níveis de fosfatase alcalina e vitamina B12. Da mesma forma, pode ocorrer transformação para outras síndromes mieloproliferativas (Szuber et al., 2020; Szuber \& Tefferi, 2018).

Quando falamos de sintomas, são bem semelhantes a LMC incluindo fadiga, perda de peso, mal-estar, fácil saciedade e dor do quadrante superior esquerdo (Melo \& Gonçalves, 2016; Szuber et al., 2020).

Já suas manifestações orais também incluem inchaço da gengiva, vermelhidão, sangramento, pequenas úlceras que podem apresentar pus ou não, mal hálito, foco séptico, palidez das mucosas, cárie, periodontite (Thomas et al., 1994; Nussbaum \& Shapira, 2011; Tangudu et al., 2018).

\subsection{Leucemia Eosinofilica Crônica (LEC)}

É uma malignidade hematológica rara com níveis elevados de eosinófilos onde é caracterizada pela presença do gene de fusão FIP1L1-PDGFRA, as características diagnósticas incluem proliferação sustentada de precursores eosinofílicos com eosinofilia marcada no sangue periférico e medulo óssea (Lee et al., 2020; Morsia et al., 2020; Ruan et al., 2020).

Devido a sua raridade, não existe consenso no tratamento, com terapêutica implementada incluindo hidroxiureia, esteroides, imatinibe, interferon e outros. Apesar desses tratamentos, duas pequenas séries de casos relataram que o LEC carregava um prognóstico ruim com sobrevida média de menos de dois anos e altas taxas de transformação leucêmica (Ruan et al., 2020).

Por alguns autores a LEC é considerado um subtipo de uma variante mieloproliferativa da síndrome hipereosinofílica, enquanto outros a consideram como uma entidade independente. Em circunstâncias normais, a LEC é caracterizada por um grave envolvimento sistêmico com expressões cutâneas sutis (Vidyadharan et al., 2016).

Por ser uma doença muito rara pouco são os casos existentes que relatam suas manifestações, mais as relatadas são ulcerações recorrentes de lábios, pênis, lesões cutâneas hiperpigmentadas e unhas distróficas com placas esbranquiçadas aderentes e crostas amareladas (Vidyadharan et al., 2016).

\subsection{Mielofibrose Idiopática Crônica (MIC)}

É uma doença mieloproliferativa de distúrbio clonal das células-tronco hematopoiéticas caracterizada por um esfregaço de sangue leucoeritroblástico com poiquilocitose em gota, esplenomegalia devido a hematopoiese extra medular, anemia de vários graus e mielofibrose da medula óssea (Rautenbach et al., 2017; Raval et al., 2019).

Nos últimos anos as investigações clínico-patológicas de MIC elucidaram a dinâmica do processo da doença, onde fizeram a investigação por biopsia sequencial de medula óssea. De acordo com os resultados derivados desses estudos, o MIC não se limita à espécie humana e a progressão da doença é gradual, assim, o amplo espectro de apresentações clínicas é refletido pela variação significativa da morfologia da medula (Chinnasami, 2017; Rautenbach et al., 2017; Kumar, 2018; Raval et al., 2019).

Após a busca pela literatura, verificou-se que não há trabalhos que mostrem alterações orais ou sintomatologia na mucosa oral.

\subsection{Policitemia Vera (PV)}

A policitemia vera é a mais comum neoplasia mieloproliferativa, possui a maior incidência de complicações tromboembólicas, que geralmente ocorrem no início da doença. A apresentação clássica de PV é caracterizada por eritrócitose, 
leucocitose e trombocitose, muitas vezes com esplenomegalia e ocasionalmente com mielofibrose, mas também pode apresentar-se como eritrócito isolada com ou sem esplenomegalia, trombocitose isolada ou leucocitose isolada, ou qualquer combinação destes (Tefferi \& Barbui 2017; Spivak, 2018).

O diagnóstico de PV muitas vezes requer a presença de uma mutação $J A K 2$, além da documentação de aumento da hemoglobina e hematócrito, a um nível limiar estabelecido pela OMS. Além disso, a avaliação morfológica da medula óssea é encorajada, a fim de distinguir PV do ET -mutado JAK2 (Barbui et al., 2017; Kvasnicka et al., 2017).

Suas características clínicas incluem um grau leve a moderada de esplenomegalia, fadiga e prurido, sintomas de hiperviscosidade, leucocitose, trombocitose, sintomas microvasculares, dores de cabeça, tontura, distúrbios visuais, dor torácica atípica, eritromelalgia, parestesia (Tefferi \& Barbui, 2017; Tefferi et al., 2018).

Já suas manifestações orais incluem petéquias na mucosa oral, hiperplasia gengival, gengivite refratária, sangramento excessivo ao menor trauma, infiltrado linfocitário, perda dentária, úlceras orais com prurido e dolorosas, pigmentação da gengiva, erosões eritâmicas, lesões hiperqueratóticas (Alshammasi et al., 2018; Messadi et al., 2019; Oueslati et al., 2020).

\subsection{Trombocitopenia Essencial (TE)}

A trombocitemia essencial é uma desordem mieloproliferativa crônica, caracterizada pela proliferação clonal de megacariócitos na medula óssea, culminando com o aumento persistente das plaquetas circulantes no sangue. Onde os níveis plaquetários encontrado na doença é acima dos valores de referência, clinicamente acompanhado de esplenomegalia e episódios trombóticos e hemorrágicos. É uma doença rara que geralmente atinge pessoas adultas de 50 e 60 anos, sendo algo incomum em crianças, que quando ocorre é assintomática (Haider et al., 2016; Sampaio et al., 2018; Tefferi \& Barbui, 2019).

Suas manifestações características compreendem cefaleia, febre, prurido, sudorese, acidente vascular cerebral, distúrbios visuais, angina, priaprismo, parestesias e eritromialgia (Haider et al., 2016), quando falamos da cavidade oral os únicos achados foram petéquias na mucosa oral e hemorragia gengival (Regezi et al., 2017; Prasad et al., 2018).

\section{Resultados e Discussão}

O presente trabalho relacionou as alterações orais causadas pelas doenças mieloproliferativas em busca de aprimorar o diagnostico diferencial este trabalho trás as principais alterações encontradas na literatura sobre as alterações bucais. Os artigos e suas respectivas abordagens nas manifestações orais estão contidos na Tabela 1. 
Tabela 1. Manifestações orais das doenças mieloproliferativas.

\begin{tabular}{|c|c|c|}
\hline Autores & Doenças & Manifestações orais \\
\hline $\begin{array}{l}\text { Boddu et al., 2017; Shimizu et al., } \\
\text { 2017; Ratre et al., 2018; Shen et al., } \\
\text { 2018; Tag-Adeen et al., } 2018\end{array}$ & Leucemia Mieloide Crônica & $\begin{array}{l}\text { Queilite angular, manchas violáceas, pápula } \\
\text { sobre o freio labial, hiperplasia fibrosa } \\
\text { inflamatória, úlcera sublingual, papila } \\
\text { incisiva hiperemiada, múltiplas petéquias e } \\
\text { descamação labial. }\end{array}$ \\
\hline $\begin{array}{l}\text { Thomas et al., 1994; Nussbaum \& } \\
\text { Shapira, 2011; Tangudu et al., } 2018\end{array}$ & Leucemia Neutrofilica Crônica & $\begin{array}{l}\text { Inchaço da gengiva, vermelhidão, } \\
\text { sangramento, pequenas úlceras que podem } \\
\text { apresentar pus ou não, mal hálito, foco } \\
\text { séptico, palidez das mucosas, cárie, } \\
\text { periodontite. }\end{array}$ \\
\hline Vidyadharan et al., 2016 & Leucemia Eosinofilica Crônica & $\begin{array}{l}\text { Ulcerações recorrentes de lábios, } \\
\text { hiperpigmentadas. }\end{array}$ \\
\hline- & Mielofibrose Idiopática Crônica & - \\
\hline $\begin{array}{l}\text { Alshammasi et al., 2018; Messadi et } \\
\text { al., 2019; Oueslati et al., } 2020\end{array}$ & Policitemia Vera & $\begin{array}{l}\text { Petéquias na mucosa oral, hiperplasia } \\
\text { gengival, gengivite refratária, sangramento } \\
\text { excessivo ao menor trauma, infiltrado } \\
\text { linfocitário, perda dentária, úlceras orais } \\
\text { com prurido e dolorosas, pigmentação da } \\
\text { gengiva, erosões eritâmicas, lesões } \\
\text { hiperqueratóticas. }\end{array}$ \\
\hline Regezi et al., 2017; Prasad et al., 2018 & Trombocitopenia Essencial & $\begin{array}{l}\text { Petéquias na mucosa oral e hemorragia } \\
\text { gengival. }\end{array}$ \\
\hline
\end{tabular}

Fonte: Autores.

Por serem raras, as doenças mieloproliferativas não são de fácil diagnostico onde suas principais alterações são a multiplicação desordenada das células mieloides, esta alteração desencadeia uma série de manifestações sistêmicas no indivíduo, onde podem evoluir para o óbito.

Estas manifestações são muito importantes nas doenças hematológicas, uma vez que é sabido o conhecimento dos sinais e sintomas é possível o diagnóstico e a prevenção das mesmas.

Macedo et al., (2017) diz que o diagnostico molecular é fundamental para fechar o diagnostico dessas doenças hematológicas, pois eles conseguem identificas rearranjo e polimorfismos característicos das doenças, podem com o conhecimento das manifestações sistêmicas e principalmente das orais, pode-se presumir e identificas precocemente essas patologias.

Já no relato de caso de Couples (2018), o exame hematológico foi de fundamental importância, pois revelou um aumento na contagem de leucócitos, incluindo principalmente formas jovens e basofilia, levando em consideração também os achados clínicos.

Outros estudos como o de Costa et al (2017), levam em consideração os aspectos clínicos, onde conhecimento das alterações hematológicas são necessárias para uma boa formação de um cirurgião dentista, uma vez que essas alterações tem suas primeiras manifestações na mucosa oral.

\section{Conclusão}

Espera-se que esta pesquisa juntamente com pesquisas futuras, possa esclarecer as alterações provocadas pelas doenças mieloproliferativas, principalmente suas manifestações na cavidade oral, para um diagnostico diferencial e precoce, onde também é evidente a necessidade de um acompanhamento multiprofissional dos pacientes acometidos por estas enfermidades. Dessa forma, há uma relação direta das alterações clínicas na cavidade oral em indivíduos com doença 
mieloproliferativa. Porém mais estudos devem ser realizados afim de melhor esclarecimentos.

\section{Referências}

Alshammasi, B., Albasry, Z., \& Meshikhes, F. (2020). Oral hyperpigmentation associated with hydroxyurea in a patient with polycythemia vera: A case report. 8(10), 1904-1909. https://doi.org/10.1002/ccr3.3012.

Barbui, T. et al. (2017). Diagnostic impact of the 2016 revised who criteria for polycythemia vera. Am. J. Hematol. 92, 417-419. https://doi.org/10.1002/ajh.24684.

Boddu P. et al. (2017) Necrotizing fungal gingivitis in a patient with acute myelogenous leukemia: Visible yet obscure. Journal of Oral and Maxillofacial Surgery, 30 (1) 50-54. https://doi.org/10.1016/j.ajoms.2017.08.007.

Braun, L. M., \& Zeiser, R. (2020). Immunotherapy in myeloproliferative diseases. Cells, 9(6), 1559. https://doi.org/10.3390/cells9061559.

Carvalho, J. S. et al. (2017). Steatosis caused by experimental periodontitis is reversible after removal of ligature in rats. Journal of Periodontal Research, 52(5), 883-892. https://doi.org/10.1111/jre.12459.

Chinnasami, D. (2017). Idiopathic myelofibrosis in pregnancy-a rare case report. University Journal of Surgery and Surgical Specialities, 2(7).

Costa, S. S., Sousa, H. R., \& Costa, I. S. (2017) O papel do cirurgião-dentista no diagnóstico precoce da leucemia e sua responsabilidade ética e legal-revisão de literatura. RBOL-Revista Brasileira de Odontologia Legal, 4(2). https://doi.org/10.21117/rbol.v4i2.82.

Couples (2018), Reproductive treatment of homosexual. diagnóstico de leucemia mieloide crônica em fase acelerada: relato de caso. Brasília Med, 55, 22-26. http://doi.org/10.5935/2236-5117.2018v55a02.

Eichstaedt, C. A. et al. (2020) Myeloproliferative diseases as possible risk factor for development of chronic thromboembolic pulmonary hypertension-A Genetic Study. International Journal of Molecular Sciences, 21(9), 3339. https://doi.org/10.3390/ijms21093339.

Estrela, C. (2018). Metodologia científica: ciência, ensino, pesquisa: Artes Médicas.

Szuber, N. et al. (2020) Chronic neutrophilic leukemia: 2020 update on diagnosis, molecular genetics, prognosis, and management. American journal of hematology, 95(2), 212-224. https://doi.org/10.1002/ajh.25688

França, L. F. C. et al. (2017). Periodontitis changes renal structures by oxidative stress and lipid peroxidation. Journal of Clinical Periodontology, 44(6), 568576. https://doi.org/10.1111/jcpe.12729.

Gomes-filho, I. S. et al. (2020). Periodontitis and respiratory diseases: A systematic review with meta-analysis. Oral diseases, 26(2), 439-446. https://doi.org/10.1111/odi.13228.

Haider, M. et al. (2016). Validation of the revised international prognostic score of thrombosis for essential thrombocythemia (IPSET-thrombosis) in 585 Mayo clinic patients. American Journal of Hematology, 91(4), 390-394. https://doi.org/10.1002/ajh.24293.

Houshmand, M. et al. (2019). Chronic myeloid leukemia stem cells. Leukemia, 33(7), 1543-1556. http://doi.org/10.1200/JCO.2008.17.5745.

Jabbour, E., \& Kantarjian, H. (2018) Chronic myeloid leukemia: 2018 update on diagnosis, therapy and monitoring. American journal of hematology, 93(3), 442-459. https://doi.org/10.1002/ajh.25011.

Kane, S. F. (2017). The effects of oral health on systemic health. Gen Dent, 65(6), 30-34. http://doi.org/10.1111/j.1834-7819.1999.tb00203.x.

Kumar, S. (2018). A case of choronic idiopathic myelofibrosis. University Journal of Medicine and Medical Specialities, 4(1).

Kvasnicka H. M. et al. (2017). European LeukemiaNet study on the reproducibility of bone marrow features in masked polycythemia vera and differentiation from essential thrombocythemia. Am. J. Hematol. 92, 1062-1067. https://doi.org/10.1002/ajh.24837.

Lago, C., \& Petroni, T. F. (2017). Fisiopatologia e diagnóstico da Leucemia Mielóide Crônica. Revista Saúde UniToledo, 1(1).

Lee, Ji-Sook et al. (2020). S100A8 and S100A9 Promote apoptosis of chronic eosinophilic leukemia cells. Frontiers in immunology, 11, 1258. https://doi.org/10.3389/fimmu.2020.01258.

Maxson, J. E., \& Tyner, J. W. (2017). Genomics of chronic neutrophilic leukemia. Blood, The Journal of the American Society of Hematology, 129(6), 715722. https://doi.org/10.1182/blood-2016-10-695981.

Melo, B. S. L., \& Gonçalves, D. S. S. (2016). Atuação do enfermeiro no tratamento da leucemia mielóide crônica. Revista Transformar, 9, 129-140.

Messadi, D. V., \& Mirowski, G. W. (2019) Oral signs of hematologic disease. In: Oral Signs of Systemic Disease. Springer, Cham. p. 25-43. https://doi.org/10.1007/978-3-030-10863-2_3.

Morsia, E. et al. (2020). WHO defined chronic eosinophilic leukemia, not otherwise specified (CEL, NOS): A contemporary series from the Mayo Clinic. American journal of hematology, 95(7), E172-E174. https://doi.org/10.1002/ajh.25811.

Nussbaum G., \& Shapira L. (2011) How has neutrophil research improved our understanding of periodontal pathogenesis? J Clin Periodontol. 38(11), 49-59. https://doi.org/10.1111/j.1600-051X.2010.01678.x. 
Oliveira, K. K. V. et al. (2015). Manifestações Orais nas Doenças Hematológicas: revisão de literatura. Revista da Universidade Vale do Rio Verde, 13(2), 216-235. http://dx.doi.org/10.5892/ruvrd.v13i1.2275.

Oueslati, Y. et al. (2020). Oral lichen planus and polycythemia: possible association. Case Reports in Dentistry, v. 2020. https://doi.org/10.1155/2020/8820114

Ouyang, Y. et al. (2017). Clinical significance of CSF3R, SRSF2 and SETBP1 mutations in chronic neutrophilic leukemia and chronic myelomonocytic leukemia. Oncotarget, 8(13), 20834. https://dx.doi.org/10.18632\%2Foncotarget.15355

Prasad, S. R. et al. (2018). Oral manifestations of myeloid neoplasms and acute leukemia-a diagnostic perspective. Hematol Transfus Int J, 6(5), 177-180. https://doi.org/10.15406/htij.2018.06.00177.

Ratre M. S. et al. (2018). Regular oral screening and vigilance: can it be a potential lifesaver?. J Indian Soc Periodontol. 22(3):171-173. https://dx.doi.org/10.4103\%2Fjisp.jisp_136_18.

Rautenbach, Y., Goddard, A., \& Clift, S. J. (2017). Idiopathic myelofibrosis accompanied by peritoneal extramedullary hematopoiesis presenting as refractory ascites in a dog. Veterinary clinical pathology, 46(1), 46-53. https://doi.org/10.1111/vcp.12430.

Raval, V., Sudana, P., \& Taraprasad. (2019). Idiopathic myelofibrosis causing inflammatory central retinal vein occlusion mimicking frosted branch angiitis. BMJ Case Reports CP, 12(6), e229865. http://dx.doi.org/10.1136/bcr-2019-229865.

Regezi, J., Sciubba, J. J., \& Jordan, R. C. K. (2017). Patologia oral: correlações clinicopatológicas. Elsevier Brasil.

Ribeiro, N. D. B. et al. (2021). Qualidade de vida de pacientes portadores de leucemia mieloide crônica tratados com mesilato de imatinibe. 2021.

Ruan, G. J. et al. (2020). A population-based study of chronic eosinophilic leukemia-not otherwise specified in the United States. American journal of hematology. https://doi.org/10.1002/ajh.25906.

Sampaio, J. A. M. et al. (2018). Leucemia mieloide aguda secundária à trombocitemia essencial na infância. Revista Científica Hospital Santa Izabel, 2(1), 3742. https://doi.org/10.35753/rchsi.v2i1.84.

Shen Y. et al. (2018). Multifocal occurrence of intraoral isolated MS in a patient without leukemic presentation: A case report and literature review. Oral Medicine. 125(3):42-48. https://doi.org/10.1016/j.oooo.2017.11.013.

Shimizu R. et al. (2017). Unusual maxillary osteoblastic and osteolytic lesions presenting as an initial manifestation of childhood acute myeloid leukemia: A case report. Quintessence. 48(2):149-153. http://doi.org/10.3290/j.qia37383.

Sossela, F. R., Zoppas, B. C. A., \& Weber, L. P. (2017). Leucemia Mieloide Crônica: aspectos clínicos, diagnóstico e principais alterações observadas no hemograma. RBAC, 49(2), 127-30. http://doi.org/10.21877/2448-3877.201700543.

Spivak, J. L. (2018). Polycythemia vera. Current treatment options in oncology, 19(2), 12. https://doi.org/10.1007/s11864-018-0529-x.

Spivak, J.L. (2017). Neoplasias mieloproliferativas. Engl. J. Med. 377, 895-896.

Szuber, N., \& Tefferi, A. (2018). Chronic neutrophilic leukemia: new science and new diagnostic criteria. Blood cancer journal, 8(2), 1-19. http://doi.org/10.1038/s41408-018-0049-8.

Tag-Adeen M. et al. (2018). An unusual presentation of adult T-cell leukemia/lymphoma. Ecancermedicalscience. 12:801. https://dx.doi.org/10.3332\%2Fecancer.2018.801.

Tangudu, B. A. et al. (2018). Oral manifestations associated with neutrophil deficiency and neutrophil disorders: A review. IAIM. 5(6): 61-71.

Tefferi, A. \& Barbui, T. (2017). Polycythemia vera and essential thrombocythemia: 2017 update on diagnosis, risk-stratification, and management. Am. $J$. Hematol. 92, 94-108. https://doi.org/10.1002/ajh.24607.

Tefferi, A., \& Barbui, T. (2019) Polycythemia vera and essential thrombocythemia: 2019 update on diagnosis, risk-stratification and management. American journal of hematology, 94(1), 133-143. https://doi.org/10.1002/ajh.25303.

Tefferi, A., Vannucchi, A. M., \& Barbui, T. (2018). Polycythemia vera treatment algorithm 2018. Blood cancer journal, 8(1), 1-7. https://doi.org/10.1038/s41408-017-0042-7.

Thomas C. H., \& Shapira, T. E. L. (1994). Van Dyke. Neutrophil defects as risk factors for periodontal disease. J Periodontol, 521-529. https://doi.org/10.1902/jop.1994.65.5s.521.

Vainchenker, W., \& Kralovics, R. (2017). Base genética e fisiopatologia molecular de neoplasias mieloproliferativas clássicas. Sangue, 129, 667-679.

Vidyadharan, S., Joseph, B., \& Nair, S. P. (2016). Chronic eosinophilic leukemia presenting predominantly with cutaneous manifestations. Indian journal of dermatology, 61(4), 437. https://dx.doi.org/10.4103\%2F0019-5154.185716.

Zhao, H., \& Deininger, M. W. (2020). Declaration of Bcr-Abl1 independence. Leukemia, p. 1-10. 